\title{
Correlation of X-ray Computed Nanotomography and Scanning Electron Microscopy Imaging of Collagen Scaffolds
}

\author{
Dominika Kalasová $^{1, *}$, Veronika Pavliňáková ${ }^{1}$, Tomáš Zikmund ${ }^{1}$, Lucy Vojtová ${ }^{1}$ and Jozef Kaiser ${ }^{1}$. \\ 1. CEITEC - Central European Institute of Technology, Brno University of Technology, Brno, Czech \\ Republic \\ * Corresponding author, dominika.kalasova@ceitec.vutbr.cz
}

Many tissue engineering approaches involve an application of 3D scaffolds. Numerous scaffolds produced from a variety of biomaterials (collagen, chitosan, elastin, etc.) are used in many fields to regenerate different tissues and organs such as bones [1] and for cartilage and cardiovascular repair [2]. As an example, the collagen is a popular extracellular matrix of molecules of connective tissues because of its abundance, biodegradability, and biocompatibility [3]. The morphology of porous scaffolds is usually observed using scanning electron microscopy (SEM). X-ray computed nanotomography (nCT) imaging is a technique enabling a 3D investigation of scaffold's structure and thus a quantification of cell's and scaffold's characteristics such as their size, 3D distribution, porosity, thickness etc. [4, 5].

In the present study, the collagen scaffold structure was observed using a nCT RIGAKU Nano3DX and a SEM Tescan MIRA3. These two machines have completely different geometrical arrangements. To be able to locate the same region of interest of the sample in both nCT and SEM measurements, a sample holder compatible with nCT and SEM had to be manufactured. Consequently, the sample didn't have to be removed from the holder during measurements on either machine. The sample holder was designed as a rounded bar split in two parts (Fig. 1). The upper part (12 mm height) allows fastening the sample to the MIRA3, whereas the entire sample holder allows fastening the sample to the Nano3DX (Fig. 2). For an easy dismantling, the upper part is connected by M2 set screw and can be demounted easily by bare hands. Both parts of the holder are made of stainless steel grade 1.4305 (AISI 303). This grade was chosen for its good temperature stability and excellent machinability.

To locate precisely the same regions of interest, e.g. cells on scaffold, it is advantageous that the marker is placed on a sample and easily recognizable in both measurement methods. A test of several markers was performed to select the best one for both nCT and SEM. Tested materials were red and silver nail polish, carbon tape, carbon tape with pieces of aluminium foil, and handle tape. All of these were applied to the collagen scaffold and imaged with a light microscope (LM), SEM and nCT (Fig. 3). nCT data were processed in VGSTUDIO MAX 3.1 software and the markers were segmented and rendered in 3D (Fig. 3 bottom left). For the comparison of 3D nCT and 2D LM and SEM, the surface of the sample was rendered in the nCT data to obtain a similar view as in the SEM and LM.

All markers containing metals (aluminium foil, handle tape) caused noticeable metal artefacts, which strongly decreased quality of nCT data (Fig. 3, nCT slice 2). Carbon tape does not have such a contrast at SEM as nail polish. Eventually, silver nail polish was chosen as the best marker, because of its easy detection and no artefacts in both nCT and SEM.

The proper matching of SEM and nCT data by the use of a sample holder and a suitable marker allows to confirm the position of the cells within the scaffold. In this way of material's determination in nCT data, it will be possible to study cell's distribution within scaffold and determine its characteristics such as 
volume, 3D distribution or amount of proliferation [6].

References:

[1] E. Prosecká et al, J. Biomed. Mater. Res. A 99A (2011) 307.

[2] Ch. Bermueller et al, Tissue Eng. Pt. A 19 (2013) 2201.

[3] J. Glowacki and S. Mizuno, Biopolymers 89 (2008) 338.

[4] K. Brockdorf et al, Microsc. Microanal. 13 (2007) 1572.

[5] R. S. Bradley et al, Macromol. Biosci. 17 (2017) 1600236.

[6] This research has been financially supported by the MEYS CR under the project CEITEC 2020 (LQ1601) and CEITEC Nano Research Infrastructure (MEYS CR, 2016-2019), and TACR (TJ01000382).

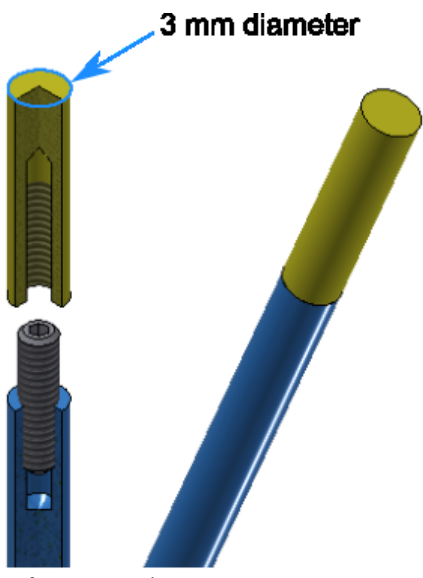

Figure. 1. SEM-nCT holder for samples. The upper (yellow) part can be unscrewed and inserted into microscope.

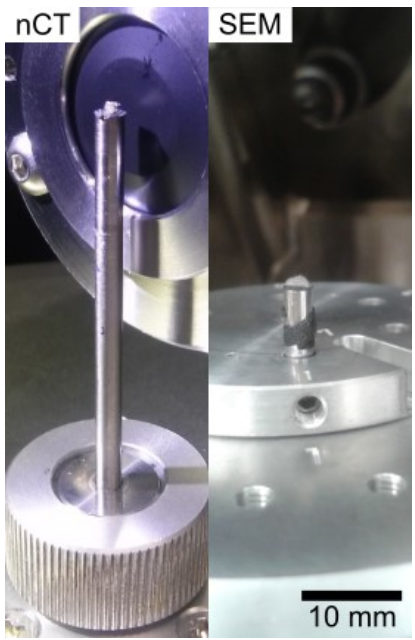

Figure. 2. SEM-nCT holder for samples in nCT and SEM.
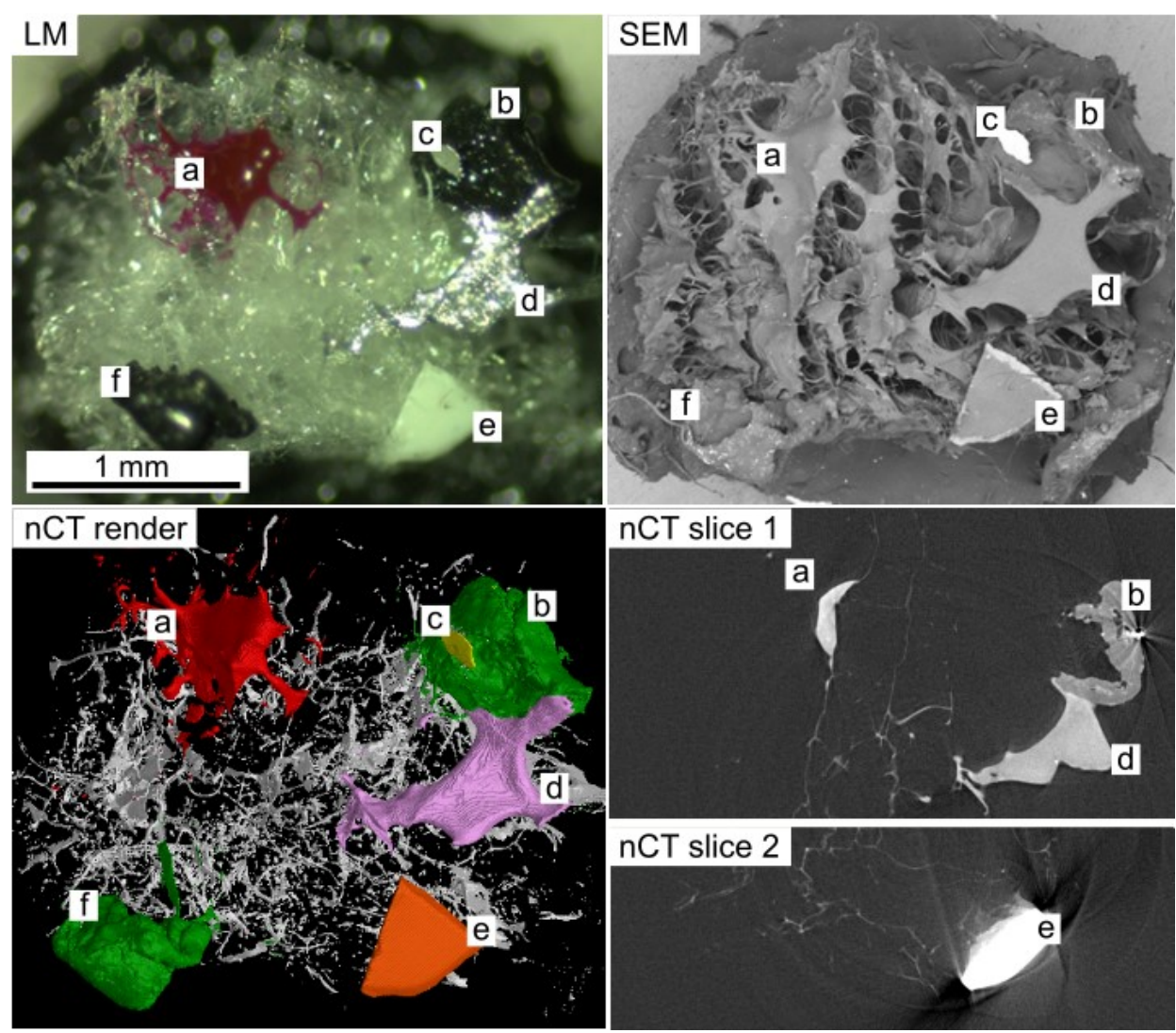

nCT slice 1

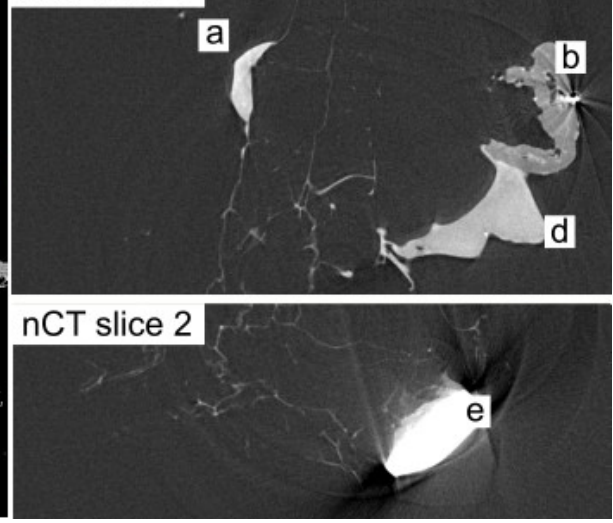

Figure. 3. Test of makers for correlation of light microscopy (LM), scanning electron microscopy (SEM) and X-ray computed nanotomography (nCT). Selected markers are applied on the collagen scaffold: a) red nail polish, b) carbon tape + c) piece of aluminum film, d) silver nail polish, e) handle tape, f) carbon tape. All images have the same scale. 